DMTCS proc. BC, 2016, 611-622

\title{
The Delta Conjecture
}

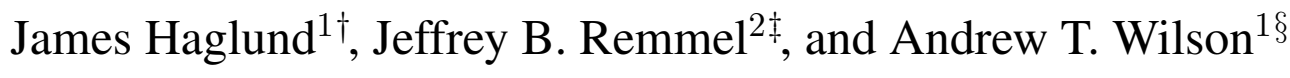 \\ ${ }^{1}$ Department of Mathematics, University of Pennsylvania \\ ${ }^{2}$ Deparment of Mathematics, UC San Diego
}

\begin{abstract}
We conjecture two combinatorial interpretations for the symmetric function $\Delta_{e_{k}} e_{n}$, where $\Delta_{f}$ is an eigenoperator for the modified Macdonald polynomials defined by Bergeron, Garsia, Haiman, and Tesler. Both interpretations can be seen as generalizations of the Shuffle Conjecture, a statement originally conjectured by Haglund, Haiman, Remmel, Loehr, and Ulyanov and recently proved by Carlsson and Mellit. We show how previous work of the second and third authors on Tesler matrices and ordered set partitions can be used to verify several cases of our conjectures. Furthermore, we use a reciprocity identity and LLT polynomials to prove another case. Finally, we show how our conjectures inspire 4-variable generalizations of the Catalan numbers, extending work of Garsia, Haiman, and the first author.

Résumé. Nous conjecturons deux interprétations combinatoires pour la fonction symétrique $\Delta_{e_{k}} e_{n}$, où $\Delta_{f}$ est un eigenoperator pour les polynômes de Macdonald modifiés d’finis par Bergeron, Garsia, Haiman, et Tesler. Les deux interprétations peuvent être considérés comme des généralisations de le Shuffle Conjecture, une déclaration à l'origine conjecturé par Haglund, Haiman, Remmel, Loehr, et Ulyanov et a récemment prouvé par Carlsson et Mellit. Nous montrons comment le travail préalable des deuxième et troisième auteurs sur les matrices Tesler et ensemble ordonné partitions peuvent être utilisés pour vérifier plusieurs cas de nos conjectures. En outre, nous utilisons une identité de réciprocité et LLT polynômes de prouver une autre affaire. Enfin, nous montrons comment nos conjectures inspirent généralisations 4 variables des nombres de Catalan, l'extension du travail de Garsia, Haiman, et le premier auteur.
\end{abstract}

Keywords. Macdonald polynomials, parking functions, Dyck paths, ordered set partitions, Tesler matrices

\section{Introduction}

While working towards a proof of the Schur positivity of Macdonald polynomials, Garsia and Haiman discovered the module of diagonal harmonics, an $\mathfrak{S}_{n}$-module that captures many of the properties of Macdonald polynomials. In [Hai02], Haiman proved that the Frobenius characteristic of the module of diagonal harmonics could be written as $\nabla e_{n}$ or $\Delta_{e_{n}} e_{n}$ for certain symmetric function operators $\nabla$ and $\Delta_{f}$ which are eigenoperators of Macdonald polynomials. Building on this work, Haiman, Loehr, Ulyanov, and the first two authors proposed a connection between $\nabla e_{n}$ and parking functions which has come to be known as the Shuffle Conjecture [HHL $\left.{ }^{+} 05\right]$. The goal of this abstract is to state and support two versions of a generalization of the Shuffle Conjecture in which $\Delta_{e_{n}} e_{n}$ is replaced by $\Delta_{e_{k}} e_{n}$ for an

\footnotetext{
†Email: jhaglundemath. upenn. edu. Partially supported by NSF grant DMS-1200296.

‡Email: jremmel@math.ucsd.edu.

$\S$ Email: andwils@math. upenn. edu. Partially supported by an NDSEG Fellowship and an NSF Postdoctoral Fellowship.
} 


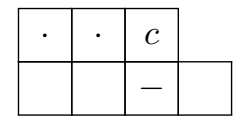

Fig. 1: This is the Young diagram (in French notation) of the partition $(4,3)$. The cell $c$ has $a^{\prime}(c)=2$ (represented by dots) and $\ell^{\prime}(c)=1$ (represented by dashes).

integer $1 \leq k \leq n$. We will also see how our generalizations tie together a wide variety of algebraic and combinatorial objects, such as parking functions, ordered set partitions, generalizations of Tesler matrices, and LLT polynomials. In this section, we provide the necessary background and then state our conjecture.

\subsection{Symmetric and Quasisymmetric Functions}

Let $\Lambda$ denote the ring of symmetric functions with coefficients in $\mathbb{Q}(q, t)$. The sets $\left\{e_{\mu}: \mu \vdash n\right\}$, $\left\{h_{\mu}: \mu \vdash n\right\},\left\{\tilde{H}_{\mu}: \mu \vdash n\right\}$ are the elementary, homogeneous, and (modified) Macdonald symmetric function bases for $\Lambda^{(n)}$, the elements of $\Lambda$ that are homogeneous of degree $n$. We use the Hall inner product on $\Lambda^{(n)}$. Given a partition $\mu \vdash n$ and a cell $c$ in the Young diagram of $\mu$ (drawn in French notation) we set $a^{\prime}(c)$ and $\ell^{\prime}(c)$ to be the number of cells in $\mu$ that are strictly to the left and strictly below $c$ in $\mu$, respectively. We define

$$
B_{\mu}(q, t)=\sum_{c \in \mu} q^{a^{\prime}(c)} t^{\ell^{\prime}(c)} \quad \text { and } \quad T_{\mu}(q, t)=\prod_{c \in \mu} q^{a^{\prime}(c)} t^{\ell^{\prime}(c)} .
$$

Given any symmetric function $f \in \Lambda$, we define operators $\Delta_{f}$ and $\Delta_{f}^{\prime}$ on $\Lambda$ by their action on the Macdonald basis:

$$
\Delta_{f} \tilde{H}_{\mu}=f\left[B_{\mu}(q, t)\right] \tilde{H}_{\mu} \quad \text { and } \quad \Delta_{f}^{\prime} \tilde{H}_{\mu}=f\left[B_{\mu}(q, t)-1\right] \tilde{H}_{\mu} .
$$

Here, we have used the notation that, for a symmetric function $f$ and a sum $A=a_{1}+\ldots+a_{N}$ of monic monomials, $f[A]$ is equal to the specialization of $f$ at $x_{1}=a_{1}, \ldots, x_{N}=a_{N}$, where the remaining variables are set equal to zero. We also set $\nabla=\Delta_{e_{n}}$ as an operator on $\Lambda^{(n)}$.

Note that, by definition, for any $1 \leq k \leq n$

$$
\Delta_{e_{k}} e_{n}=\Delta_{e_{k}+e_{k-1}}^{\prime} e_{n}=\Delta_{e_{k}}^{\prime} e_{n}+\Delta_{e_{k-1}}^{\prime} e_{n}
$$

Furthermore, for any $k>n, \Delta_{e_{k}} e_{n}=\Delta_{e_{k-1}}^{\prime} e_{n}=0$. Therefore $\Delta_{e_{n}} e_{n}=\Delta_{e_{n-1}}^{\prime} e_{n}$.

The ring of quasisymmetric functions consists of the formal power series in variables $x_{1}, x_{2}, x_{3}, \ldots$ that are translation invariant, i.e. the coefficient of $x_{1}^{\alpha_{1}} \ldots x_{n}^{\alpha_{n}}$ is equal to the coefficient of $x_{i_{1}}^{\alpha_{1}} \ldots x_{i_{n}}^{\alpha_{n}}$ for any composition $\alpha \vDash n$ and positive integers $i_{1}<i_{2} \ldots<i_{n}$. We will primarily use the monomial basis $\left\{M_{\alpha}: \alpha \vDash n\right\}$ for the quasisymmetric functions that are homogeneous of degree $n$, where $\alpha$ is a composition whose sum is $n$. Although not every function we define in our conjecture is obviously symmetric, it is clear that each function is quasisymmetric, and thus can be expanded in the monomial quasisymmetric basis.

\subsection{Labeled Dyck Paths}

To state our conjectures, we consider objects which we call labeled Dyck paths. A Dyck path of order $n$ is a lattice path from $(0,0)$ to $(n, n)$ consisting of north and east steps that remains weakly above the line 


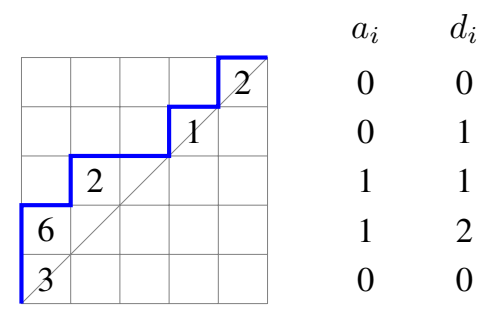

Fig. 2: A labeled Dyck path $P \in \mathcal{L D}_{5}$ with area $(P)=2, \operatorname{dinv}(P)=4$, and $\operatorname{Val}(P)=\{4,5\}$.

$y=x$, which is sometimes called the diagonal, main diagonal, or 0-diagonal. To obtain a labeled Dyck path, we label the north steps of a Dyck path with (not necessarily unique) positive integers such that the labels strictly increase while ascending each column. We denote the Dyck paths and labeled Dyck paths of order $n$ by $\mathcal{D}_{n}$ and $\mathcal{L} \mathcal{D}_{n}$, respectively. Often, we will use the notation $D(P)$ to denote the underlying Dyck path of a labeled Dyck path $P$. Labeled Dyck paths are sometimes called (word) parking functions, since there is a classical bijection between the two classes of objects.

Given a Dyck path $D \in \mathcal{D}_{n}$, we number the rows of $D$ with $1,2, \ldots, n$ from bottom to top. Then, for each row $i$, we set the area of the row $i$, written $a_{i}(D)$, to be the number of full squares between $P$ and the diagonal. A labeled Dyck path $P$ inherits the values $a_{i}(P)$ from its underlying Dyck path $D(P)$. We also set

$$
\begin{aligned}
d_{i}(P) & =\left|\left\{i<j \leq n: a_{i}(P)=a_{j}(P), \ell_{i}(P)<\ell_{j}(P)\right\}\right| \\
& +\left|\left\{i<j \leq n: a_{i}(P)=a_{j}(P)+1, \ell_{i}(P)>\ell_{j}(P)\right\}\right| .
\end{aligned}
$$

where $\ell_{i}(P)$ is the label in the $i$ th row of $P$. These are the primary and secondary diagonal inversions beginning in row $i$, respectively. The area and dinv statistics are defined by area $(P)=\sum_{i=1}^{n} a_{i}(P)$ and $\operatorname{dinv}(P)=\sum_{i=1}^{n} d_{i}(P)$.

The contractible valleys of $P$ are

$$
\begin{aligned}
\operatorname{Val}(P) & =\left\{2 \leq i \leq n: a_{i}(P)<a_{i-1}(P)\right\} \\
& \cup\left\{2 \leq i \leq n: a_{i}(P)=a_{i-1}(P), \ell_{i}(P)>\ell_{i-1}(P)\right\}
\end{aligned}
$$

Visually, these are the rows $i$ that are immediately preceded by an east step and, if we were to remove this east step and shift everything beyond it one step to the west, the resulting labeled path would still have increasing labels in its columns. For the parking function depicted in Figure 2, 3 is not a contractible valley because removing the east step that starts row 3 would result in a 2 above a 6 , so that column's labels would no longer increase from bottom to top. However, rows 4 and 5 are contractible valleys.

\subsection{The Delta Conjecture}

Finally, by $x^{P}$ we mean the monomial $\prod_{i=1}^{n} x_{\ell_{i}(P)}$ and for any polynomial $f(z)$ we use $\left.f(z)\right|_{z^{k}}$ to denote the coefficient of $z^{k}$ in $f$. We use the standard notation for $q$ - and $q, t$-integers and binomial coefficients. With these definitions in hand, we can state our main conjecture, which we call the Delta Conjecture. Sometimes we will refer to (2) as the Rise Version and (3) as the Valley Version of the Delta Conjecture. 


\begin{tabular}{|c|c|c|c|}
\hline Conditions & LHS of 2 & RHS of 2 & RHS of 3 , \\
\hline Coefficient of $M_{1^{n}}$ at $q=0$ & |Wil15c| & Section 2 & Section 2 \\
\hline Coefficient of $M_{1^{n}}$ at $t=0$ & Wil15c & Section $\overline{\overline{2}}$ & Section $\overline{2}$ \\
\hline Coefficient of $M_{1^{n}}$ at $q=1$ & Wil15c & [Wil15c] & $?$ \\
\hline$\left\langle\cdot, e_{n-d} h_{d}\right\rangle, t=1 / q$ & Section 3 & [Wil15b] & [Wil15b] \\
\hline$\left.{ }_{d} h_{d}\right\rangle, t=1 / q$ & Section $\overline{3}$ & Wil15b & [Wil15b] \\
\hline$k=1$ & Section 4 & Section 4 & ? \\
\hline
\end{tabular}

Fig. 3: This table summarizes cases of the Delta Conjecture that have been proved. We provide a section number if the case is discussed in this abstract and a citation otherwise. Question marks indicate cases which have not been proved.

Conjecture 1.1 (Delta Conjecture) For any integers $n>k \geq 0$,

$$
\begin{aligned}
& \Delta_{e_{k}}^{\prime} e_{n}=\left.\sum_{P \in \mathcal{L} \mathcal{D}_{n}} q^{\operatorname{dinv}(P)} t^{\operatorname{area}(P)} \prod_{i: a_{i}(P)>a_{i-1}(P)}\left(1+z / t^{a_{i}(P)}\right) x^{P}\right|_{z^{n-k-1}} \\
& \Delta_{e_{k}}^{\prime} e_{n}=\left.\sum_{P \in \mathcal{L} \mathcal{D}_{n}} q^{\operatorname{dinv}(P)} t^{\operatorname{area}(P)} \prod_{i \in \operatorname{Val}(P)}\left(1+z / q^{d_{i}(P)+1}\right) x^{P}\right|_{z^{n-k-1}} .
\end{aligned}
$$

Equivalently, we can replace the left-hand side with $\Delta_{e_{k}} e_{n}$ for integers $n \geq k \geq 0$, multiply both righthand sides by $(1+z)$, and then take the coefficient of $z^{n-k}$.

Most of the remainder of the abstract is devoted to this conjecture. We summarize the current status of this progress in Figure 3 . Section 2 connects previous work of the second and third authors on ordered set partitions and Tesler matrices in [RW15, Wil15c] to cases of the Delta Conjecture. Section 3 provides a plethystic formula for $\Delta_{e_{k}} e_{n}$ at $t=1 / q$ and uses a result of Garsia, Leven, Wallach, and Xin [GLWX15] to give Schur positivity in this case. In Section 4, we use a reciprocity identity and LLT polynomials to sketch a proof of the $k=1$ case of (2). Section 5 contains a variation of the Delta Conjecture that involves new 4-variable Catalan polynomials. Finally, we use Section 6 to outline some of the major open problems related to the Delta Conjecture. In particular, Carlsson and Mellit [CM15] have recently announced a proof of the Shuffle Conjecture, which is equivalent to the $k=n-1$ case of the Delta Conjecture. We briefly discuss how their ideas might be used in our setting.

\section{Ordered Set Partitions and the $q=0$ or $t=0$ Cases}

In this section, we show how previous work of the second and third authors in [RW15, Wil15c] can be used to prove the following result.

Theorem 2.1 The coefficients of $M_{1^{n}}$ in the monomial quasisymmetric function expansion of the following are all equal:

$$
\begin{aligned}
& \operatorname{Rise}_{n, k}(x ; q, 0), \operatorname{Rise}_{n, k}(x ; 0, q), \operatorname{Val}_{n, k}(x ; q, 0), \\
& \operatorname{Val}_{n, k}(x ; 0, q),\left.\quad \Delta_{e_{k}}^{\prime} e_{n}\right|_{t=0},\left.\quad \Delta_{e_{k}}^{\prime} e_{n}\right|_{q=0, t=q} .
\end{aligned}
$$


In order to prove Theorem 2.1. we show how labeled Dyck paths are related to objects called ordered set partitions when $q$ or $t$ equals zero. The ordered set partitions of order $n$ with $k$ blocks are partitions of the set $\{1,2, \ldots, n\}$ into $k$ subsets (called blocks) with some order on the blocks. We write this set as $\mathcal{O} \mathcal{P}_{n, k}$. For example, $24|3| 15$ is an element of $\mathcal{O P}_{5,3}$, where we have listed each block in increasing order and used bars to separate blocks.

More generally, given a composition $\alpha$ of length $n$, the ordered multiset partitions $\mathcal{O P}_{\alpha, k}$ are the partitions of the multiset $\left\{i^{\alpha_{i}}: 1 \leq i \leq n\right\}$ into $k$ ordered blocks, each of which is a set. For example, $23|13| 24$ is in $\mathcal{O P} \mathcal{P}_{\alpha, 3}$ for $\alpha=\{1,2,2,1\}$. In [Wil15c], the third author showed that

$$
\left.\Delta_{e_{k}}^{\prime} e_{n}\right|_{M_{1} n, t=0}=\left.\Delta_{e_{k}}^{\prime} e_{n}\right|_{M_{1}, q=0, t=q}=\sum_{\pi \in \mathcal{O P}_{n, k+1}} q^{\operatorname{inv}(\pi)}
$$

where $\operatorname{inv}(\pi)$ counts the number of pairs $a>b$ such that $a$ 's block is strictly to the left of $b$ 's block in $\pi$ and $b$ is minimal in its block in $\pi$. For example, $15|23| 4$ has two inversions, between the 5 and the 2 and the 5 and the 4 . We claim that setting one of $q$ or $t$ equal to zero in our combinatorial interpretations also yields a sum involving ordered partitions. To make this more precise, we define three more statistics on ordered multiset partitions.

First, given some $\pi \in \mathcal{O} \mathcal{P}_{\alpha, k}$ we number $\pi$ 's blocks $\pi_{1}, \pi_{2}, \ldots, \pi_{k}$ from left to right. Let $\pi_{i}^{h}$ be the $h$ th smallest element in $\pi_{i}$, beginning at $h=0$. Then the diagonal inversions of $\pi$, written $\operatorname{Dinv}(\pi)$, are the triples

$$
\left\{(h, i, j): 1 \leq i<j \leq k, \pi_{i}^{h}>\pi_{j}^{h}\right\} \cup\left\{(h, i, j): 1 \leq i<j \leq k, \pi_{i}^{h}<\pi_{j}^{h+1}\right\} .
$$

The triples of the first type are primary diagonal inversions, and the triples of the second type are secondary diagonal inversions. We set $\operatorname{dinv}(\pi)$ to be the cardinality of $\operatorname{Dinv}(\pi)$.

To define the major index of $\pi$, we consider the permutation $\sigma=\sigma(\pi)$ obtained by writing each block of $\pi$ in decreasing order. Then we recursively form a word $w$ by setting $w_{0}=0$ and $w_{i}=$ $w_{i-1}+\chi\left(\sigma_{i}\right.$ is minimal in its block in $\left.\pi\right)$. Then we set

$$
\operatorname{maj}(\pi)=\sum_{i: \sigma_{i}>\sigma_{i+1}} w_{i}
$$

Finally, we define the minimum major index of $\pi$ as follows. We begin by writing the elements of $\pi_{k}$ in increasing order from left to right. Then, recursively for $i=k-1$ to 1 , we choose $r$ to be the largest element in $\pi_{i}$ that is less than or equal to the leftmost element in $\pi_{i+1}$, as previously recorded. If there is no such $r$, we write $\pi_{i}$ in increasing order. If there is such an $r$, beginning with $\pi_{i}$ in increasing order, we cycle its elements until $r$ is the rightmost element in $\pi_{i}$. We write down $\pi_{i}$ in this order. We continue this process until we have processed each block of $\pi$. For example, consider the ordered multiset permutation $\pi=13|23| 14 \mid 234$. Processing the blocks of $\pi$ from right to left, we obtain 312341234 . We consider the result as a permutation, which we denote $\tau=\tau(\pi)$, and define

$$
\operatorname{minimaj}(\pi)=\sum_{i: \tau_{i}>\tau_{i+1}} i
$$

i.e. the major index of the permutation $\tau$. The name minimaj comes from the fact that $\operatorname{minimaj}(\pi)$ is equal to the minimum major index achieved by any permutation that can be obtained by permuting elements within the blocks of $\pi$. 
Proposition 2.1 For any composition $\alpha$

$$
\begin{aligned}
\left.\operatorname{Rise}_{n, k}(x ; q, 0)\right|_{M_{\alpha}} & =\sum_{\pi \in \mathcal{O} \mathcal{P}_{\alpha, k+1}} q^{\operatorname{dinv}(\pi)} \\
\left.\operatorname{Rise}_{n, k}(x ; 0, q)\right|_{M_{\alpha}} & =\sum_{\pi \in \mathcal{O} \mathcal{P}_{\alpha, k+1}} q^{\operatorname{maj}(\pi)} \\
\left.\operatorname{Val}_{n, k}(x ; q, 0)\right|_{M_{\alpha}} & =\sum_{\pi \in \mathcal{O} \mathcal{P}_{\alpha, k+1}} q^{\operatorname{inv}(\pi)} \\
\left.\operatorname{Val}_{n, k}(x ; 0, q)\right|_{M_{\alpha}} & =\sum_{\pi \in \mathcal{O} \mathcal{P}_{\alpha, k+1}} q^{\operatorname{minimaj}(\pi)} .
\end{aligned}
$$

The proof involves analyzing the labeled Dyck paths that contribute to each left-hand side when $q$ or $t$ equals zero. In particular, we give bijections between contributing labeled Dyck paths and ordered set partitions, and these bijections lead to each of the four statistics.

The main result in [Wil15a] is that the right-hand sides of (5), (6), and (7) are all equal. In recent work, [HRRW] shows the right-hand side of $[8]$ is equal to the other three right-hand sides. Together with the results in $[\overline{W i l 15 \mathrm{c}}]$, this concludes the proof of Theorem 2.1 .

\section{Results at $t=1 / q$}

In this section, we consider the special case $t=1 / q$. As in the Shuffle Conjecture, this case is much more approachable from the symmetric function point of view than the general setting. In particular, it is not difficult to obtain a plethystic formula for $\Delta_{e_{k}} e_{n}$ at $t=1 / q$. We state the plethystic formula below and then use it to show that $\Delta_{e_{k}} e_{n}$ is Schur positive at $t=1 / q$ up to a power of $q$.

Theorem 3.1 For any symmetric function $f \in \Lambda^{(k)}$,

$$
\left.\Delta_{f} e_{n}\right|_{t=1 / q}=\frac{f\left[[n]_{q}\right] e_{n}\left[X[k+1]_{q}\right]}{q^{k(n-1)}[k+1]_{q}} .
$$

The proof uses Cauchy's Formula along with the fact that Macdonald polynomials at $t=1 / q$ are closely related to Schur functions. From Theorem 3.1 it is easy to compute

$$
\left.\Delta_{e_{k}} e_{n}\right|_{t=1 / q}=\frac{q^{\left(\begin{array}{c}
k \\
2
\end{array}\right)-k(n-1)}}{[k+1]_{q}}\left[\begin{array}{l}
n \\
k
\end{array}\right]_{q} e_{n}\left[X[k+1]_{q}\right] .
$$

In [Wil15b], the third author uses this formula along with combinatorial recursions to prove both versions of the Shuffle Conjecture after setting $t=1 / q$ and taking the inner product with $e_{n-d} h_{d}$ or $h_{n-d} h_{d}$ for any nonnegative integer $d$. We can also use Theorem 3.1 along with a recent result of Garsia, Leven, Wallach, and Xin [GLWX15] to give a Schur positivity result for our symmetric function at $t=1 / q$.

Corollary 3.1 The coefficient of any Schur function in the Schur expansion of $\left.q^{k(n-1)-\left(\begin{array}{c}k \\ 2\end{array}\right)} \Delta_{e_{k}} e_{n}\right|_{t=1 / q}$ is a polynomial in $q$ and $t$ with nonnegative integer coefficients. 


\section{Proof of the Rise Version at $k=1$}

In this section, we sketch the proof of the following special case of the Rise Version of the Delta Conjecture.

Theorem 4.1 For any positive integer $n$,

$$
\begin{aligned}
\Delta_{e_{1}} e_{n} & =\operatorname{Rise}_{n, 0}(x ; q, t)+\operatorname{Rise}_{n, 1}(x ; q, t) \\
& =\sum_{m=0}^{\lfloor n / 2\rfloor} s_{2^{m}, 1^{n-2 m}} \sum_{p=m}^{n-m}[p]_{q, t} .
\end{aligned}
$$

This verifies (2) from Conjecture 1.1 for $k=1$.

We deal with the symmetric function component of Theorem 4.1 in Subsection 4.1 and the combinatorial component in Subsection 4.2 .

\subsection{The Symmetric Side}

In this subsection, we prove the "symmetric side" of Theorem 4.1 restated below.

Proposition 4.1 For any positive integer $n$,

$$
\Delta_{e_{1}} e_{n}=\sum_{m=0}^{\lfloor n / 2\rfloor} s_{2^{m}, 1^{n-2 m}} \sum_{p=m}^{n-m}[p]_{q, t} .
$$

Our main tool will be the following reciprocity rule for the operator $\Delta$, which was proved by the first author as Corollary 2 in [Hag04].

Lemma 4.1 (Corollary 2 in [Hag04]) For positive integers $d, n$ and any symmetric function $f \in \Lambda^{(n)}$,

$$
\left\langle\Delta_{e_{d-1}} e_{n}, f\right\rangle=\left\langle\Delta_{\omega f} e_{d}, h_{d}\right\rangle .
$$

We set $d=2$ and $f=s_{\lambda}$ for $\lambda \vdash n$, since taking the inner product of a symmetric function with $s_{\lambda}$ yields the coefficient of $s_{\lambda}$ in the Schur expansion of that symmetric function. Lemma 4.1 implies that

$$
\left\langle\Delta_{e_{1}} e_{n}, s_{\lambda}\right\rangle=\left\langle\Delta_{s_{\lambda^{\prime}}} e_{2}, h_{2}\right\rangle .
$$

We can compute the right-hand side by hand. First, we expand $e_{2}$ into the modified Macdonald polynomial basis:

$$
e_{2}=\frac{1}{t-q} \tilde{H}_{1,1}-\frac{1}{t-q} \tilde{H}_{2}
$$

Then we apply the operator $\Delta_{s_{\lambda^{\prime}}}$.

$$
\Delta_{s_{\lambda}} e_{2}=\frac{s_{\lambda^{\prime}}[1+t]}{t-q} \tilde{H}_{1,1}-\frac{s_{\lambda^{\prime}}[1+q]}{t-q} \tilde{H}_{2} .
$$


Now we take the inner product with $h_{2}$, yielding

$$
\left\langle\Delta_{s_{\lambda^{\prime}}} e_{2}, h_{2}\right\rangle=\frac{s_{\lambda^{\prime}}[1+t]-s_{\lambda^{\prime}}[1+q]}{t-q} .
$$

It is already clear that the above expression is a polynomial in $q$ and $t$. Moreover, for any monomial $u$ the principal specialization $s_{\lambda^{\prime}}[1+u]$ is equal to the sum $\sum_{T} u^{\# 2}$ 's in $T$ over all semi-standard tableaux $T$ of shape $\lambda^{\prime}$ filled with 1's and 2's. This sum is zero if $\lambda^{\prime}$ has more than two rows, so we can restrict our attention to $\lambda^{\prime}=(n-m, m)$ for some integer $0 \leq m \leq\lfloor n / 2\rfloor$. For such a tableaux $T$ of shape $(n-m, m)$, it is clear that the first $m$ entries in the first row of $T$ must be 1's and all entries in the second row of $T$ must be 2's. Of the remaining $n-2 m$ entries, we are free to choose an integer $0 \leq i \leq n-2 m$ such that the left $i$ entries are 1's and the right $n-2 m-i$ entries are 2's. Hence

$$
s_{n-m, m}[1+u]=\sum_{p=m}^{n-m} u^{p}
$$

Since $(n-m, m)^{\prime}=\left(2^{m}, 1^{n-2 m}\right)$, we have

$$
\left\langle\Delta_{e_{1}} e_{n}, s_{2^{m}, 1^{n-2 m}}\right\rangle=\frac{\sum_{p=m}^{n-m} t^{p}-q^{p}}{t-q}=\sum_{p=m}^{n-m}[p]_{q, t}
$$

which proves Proposition 4.1 .

In theory, this method can be used to compute $\Delta_{e_{k}} e_{n}$ for any fixed value of $k$. For example, $\left\langle\Delta_{e_{2}} e_{n}, s_{\lambda}\right\rangle$ equals

$$
\frac{\left(t-q^{2}\right) s_{\lambda^{\prime}}\left[1+t+t^{2}\right]-(q+t+1)(t-q) s_{\lambda^{\prime}}[1+q+t]+\left(t^{2}-q\right) s_{\lambda^{\prime}}\left[1+q+q^{2}\right]}{(t-q)\left(t^{2}-q\right)\left(t-q^{2}\right)}
$$

which is clearly a polynomial in $q$ and $t$. Unfortunately, it is not clear why the resulting expression should be a positive polynomial in $q$ and $t$, and this becomes more and more of a problem as $k$ grows.

\subsection{The Combinatorial Side}

In this subsection, we sketch a proof of the following proposition, completing the proof of Theorem 4.1 .

Proposition 4.2 For any positive integer $n$,

$$
\operatorname{Rise}_{n, 0}(x ; q, t)+\operatorname{Rise}_{n, 1}(x ; q, t)=\sum_{m=0}^{\lfloor n / 2\rfloor} s_{2^{m}, 1^{n-2 m}} \sum_{p=m}^{n-m}[p]_{q, t} .
$$

First, we note that $\operatorname{Rise}_{n, 0}(x ; q, t)=e_{n}=s_{1^{n}}$, which accounts for the $m=p=0$ term above. We will need to work harder to expand $\operatorname{Rise}_{n, 1}(x ; q, t)$. We note that $\operatorname{Rise}_{n, k}(x ; q, t)$ is closely related to the LLT polynomials of [LLT97]. There are many benefits of this connection between $\operatorname{Rise}_{n, k}(x ; q, t)$ and LLT polynomials. The first is that LLT polynomials are known to be symmetric, which implies that $\operatorname{Rise}_{n, k}(x ; q, t)$ is symmetric. On the other hand, we are still unable to prove that $\operatorname{Val}_{n, k}(x ; q, t)$ is symmetric. 
More pertinent to our current case, much is known about the LLT polynomials that appear in $\operatorname{Rise}_{n, k}(x ; q, t)$. In particular, Carré and Leclerc [CL95] showed how to expand "2-column" LLT polynomials into Schur functions. In the full version of this abstract, we leverage this information to complete the proof of Theorem 4.1

\section{4-Variable Catalan Polynomials}

It is well-known [Hag08] that taking the inner product with $e_{n}$ corresponds to removing the labels from a labeled Dyck path. Thus, the Delta Conjecture implies the following conjecture.

$$
\begin{aligned}
\left\langle\Delta_{e_{k}}^{\prime} e_{n}, e_{n}\right\rangle & =\left.\sum_{D \in \mathcal{D}_{n}} q^{\operatorname{dinv}(D)} t^{\operatorname{area}(D)} \prod_{a_{i}(D)>a_{i-1}(D)}\left(1+z / t^{a_{i}(D)}\right)\right|_{z^{n-k-1}} \\
& =\left.\sum_{D \in \mathcal{D}_{n}} q^{\operatorname{dinv}(D)} t^{\operatorname{area}(D)} \prod_{i \in \operatorname{Val}(D)}\left(1+z / q^{d_{i}(D)+1}\right)\right|_{z^{n-k-1}} .
\end{aligned}
$$

The statistics $a_{i}$ and area are defined by simply ignoring the labels of a labeled Dyck path. $d_{i}$ and dinv are obtained by labeling the Dyck path $D$ so that it strictly decreases in reading order. as in [Hag08]. This conjecture is a generalization of the $q, t$-Catalan theorem proved by Garsia and the first author [GH03].

Given the combinatorial interpretations in the Delta Conjecture, it is natural to wonder if we can combine them in a way that includes both products. Unfortunately, the polynomial

$$
\sum_{P \in \mathcal{L} \mathcal{D}_{n}} q^{\operatorname{dinv}(P)} t^{\operatorname{area}(P)} \prod_{i \in \operatorname{Val}(P)}\left(1+z / q^{d_{i}(P)+1}\right) \prod_{a_{i}(P)>a_{i-1}(P)}\left(1+w / t^{a_{i}(P)}\right) x^{P}
$$

is not symmetric. However, we do seem to obtain an interesting polynomial in the Catalan case. We set

$$
\begin{aligned}
\operatorname{Cat}_{n}(q, t, z, w)= & \sum_{D \in \mathcal{D}_{n}} q^{\operatorname{dinv}(D)} t^{\operatorname{area}(D)} \prod_{i \in \operatorname{Val}(D)}\left(1+z / q^{d_{i}(D)+1}\right) \\
& \times \prod_{a_{i}(D)>a_{i-1}(D)}\left(1+w / t^{a_{i}(D)}\right) .
\end{aligned}
$$

As in the Delta Conjecture, we have a second (conjecturally equivalent) combinatorially defined polynomial. Given a Dyck path $D$, we set $b_{i}(D)$ to be the number of diagonal inversions between the $i$ th label in reading order and labels that precede it in reading order, the reading order processes the columns from largest $a_{i}$ to smallest and right to left. We define

$$
\begin{aligned}
\overline{\operatorname{Cat}}_{n}(q, t, z, w)= & \sum_{D \in \mathcal{D}_{n}} q^{\operatorname{dinv}(D)} t^{\operatorname{area}(D)} \prod_{b_{i}(D)>b_{i-1}(D)}\left(1+z / q^{b_{i}(D)}\right) \\
& \times \prod_{a_{i}(D)>a_{i-1}(D)}\left(1+w / t^{a_{i}(D)}\right) .
\end{aligned}
$$

We call these Catalan polynomials because $\operatorname{Cat}_{n}(1,1,0,0)=\overline{\operatorname{Cat}}_{n}(1,1,0,0)=\frac{1}{n+1}\left(\begin{array}{c}2 n \\ n\end{array}\right)$, the usual Catalan number. Furthermore, we conjecture that these two polynomials are equal and that they are connected to the delta operators as follows. 
Conjecture 5.1 ( $q, t, z, w$-Catalan Conjecture)

$$
\begin{aligned}
\left.\operatorname{Cat}_{n}(q, t, z, w)\right|_{z^{k} w^{\ell}} & =\left.\overline{\operatorname{Cat}}_{n}(q, t, z, w)\right|_{z^{k} w^{\ell}} \\
& =\left\langle\Delta_{h_{k}} \nabla e_{n-k}, s_{\ell+1,1^{n-k-\ell-1}}\right\rangle \\
& =\left\langle\Delta_{h_{k}} \Delta_{e_{n-k-\ell-1}}^{\prime} e_{n-k}, e_{n-k}\right\rangle .
\end{aligned}
$$

Furthermore, each of these expressions is $k, \ell$-symmetric.

We note that the equality of the right-hand sides of (22) and (23) would follow from the Rise Version of the Delta Conjecture. This is because rows with $b_{i}>b_{i-1}$ are peaks, and turning these peaks into diagonal steps corresponds to the diagonal inversion statistic for Dyck paths in [Hag04]. It is also well-known that taking the inner product with a Schur function of hook shape yields Schröder paths [Hag04].

Proposition 5.1 For integers $m \geq k>0$, a symmetric function $f \in \Lambda^{(m)}$, and any operator $\Gamma$ defined by $\Gamma \tilde{H}_{\mu}=g_{\mu} \tilde{H}_{\mu}$ for some $g_{\mu} \in \mathbb{Q}(q, t)$, we have

$$
\left\langle\Gamma \nabla f, s_{k+1,1^{m-k-1}}\right\rangle=\left\langle\Gamma \Delta_{e_{m-k-1}}^{\prime} f, e_{m}\right\rangle .
$$

Setting $m=n-\ell$ and $\Gamma=\Delta_{h_{\ell}}$ shows the desired equality of symmetric functions in the various conjectures in this section. It is still a mystery why these symmetric functions are $k, \ell$-symmetric and how they can be connected to either of the $q, t, z, w$-Catalan polynomials. It is possible that special cases of these conjectures can be proved in a manner analogous to the special cases proved for the Delta Conjecture; we have not explored these issues at this point. The only progress we have been able to make so far is to prove (somewhat complicated) recursions for refined versions of the combinatorial polynomials appearing in Conjecture 5.1, which we will include in the full version of this abstract.

\section{Open Problems}

In this section, we describe a few open problems related to the Delta Conjecture. These accompany the problems of studying the minimaj statistic, mentioned at the end of Section 2 , and of proving cases of the $q, t, z, w$-Catalan Conjecture, as described in Section 5 .

\subsection{Schröder Paths and 1,2-Labeled Dyck Paths}

In [Wil15b], the third author develops recursions for the polynomials $\left\langle\operatorname{Rise}_{n, k}(x ; q, t), f\right\rangle$ and $\left\langle\operatorname{Val}_{n, k}(x ; q, t), f\right\rangle$ for symmetric functions $f$ of the form $h_{d} e_{n-d}$ or $h_{d} h_{n-d}$. He then uses these recursions along with various results about $q$-binomial coefficients to resolve the Delta Conjecture in the case where we take the inner product with $f$ on both sides and set $t=1 / q$. It would be nice to remove the $t=1 / q$ condition for this result, since this would yield a complete analog of the first author's results on the Shuffle Conjecture in [Hag04]. The main obstruction at this point is the symmetric function side.

Problem 6.1 Find recursions for the polynomials $\left\langle\Delta_{e_{k}} e_{n}, h_{d} e_{n-d}\right\rangle$ and

$\left\langle\Delta_{e_{k}} e_{n}, h_{d} h_{n-d}\right\rangle$ similar to the recursions obtained for the $k=n$ case in [Hag04]. 


\subsection{Symmetry of $\operatorname{Val}_{n, k}(x ; q, t)$}

We mentioned in Subsection 4.1 that, due to the connection with LLT polynomials, we know that $\operatorname{Rise}_{n, k}(x ; q, t)$ is symmetric. On the other hand, we have no such result for $\operatorname{Val}_{n, k}(x ; q, t)$. In fact, we have observed that restricting the definition of $\operatorname{Val}_{n, k}(x ; q, t)$ to labelings of a fixed Dyck path does not always yield a symmetric function, which is in stark contrast to the $\operatorname{Rise}_{n, k}(x ; q, t)$ case. This implies that the following problem could be quite difficult.

Problem 6.2 Prove $\operatorname{Val}_{n, k}(x ; q, t)$ is a symmetric function, possibly by connecting it to (generalized?) LLT polynomials.

The only partial results we have in this direction are that $\operatorname{Val}_{n, k}(x ; q, 0)$ (due to Proposition 2.1) and $\operatorname{Val}_{n, k}(x ; 1, t)=\operatorname{Rise}_{n, k}(x ; 1, t)$ are symmetric.

\subsection{Generalizations}

There are various ways one could generalize the Shuffle Conjecture. For example, we could replace $e_{k}$ by a general elementary symmetric function $e_{\lambda}$ or even a general symmetric function $f$. This would generalize the Fuss extension of the Shuffle Conjecture [HHL ${ }^{+}$05]. Alternatively, one could replace $e_{n}$ with $p_{n}$, which would hopefully have some relationship to the set of all lattice paths from $(0,0)$ to $(n, n)$, as developed for the Shuffle Conjecture in [LW07]. We would also like to develop a concrete connection between the delta operator and the Rational Shuffle Conjectures of [BGLX14] apart from the $t=1 / q$ result mentioned in Section 3. Finally, it would be quite interesting if one could find an extension of the module of diagonal harmonics with Frobenius characteristic equal to $\Delta_{e_{k}} e_{n}$.

\subsection{Towards a Proof of the Delta Conjecture}

Finally, we would be remiss if we did not mention the recent preprint of Carlsson and Mellit [CM15] which contains a proof of the Compositional Shuffle Conjecture, and therefore the $k=n-1$ case of the Delta Conjecture. It is quite possible that their proof could be adjusted to prove the Delta Conjecture, although it seems like this adjustment must be nontrivial. We are investigating ways to generalize the key recursions in [CM15] in order to apply them to the Rise Version of the Delta Conjecture. At the very least, this is a promising development towards a proof of the Delta Conjecture.

\section{References}

[BGLX14] F. Bergeron, A. Garsia, E. Leven, and G. Xin. Compositional $(k m, k n)$-shuffle conjectures. arXiv:1404.4616, April 2014.

[CL95] C. Carré and B. Leclerc. Splitting the square of a Schur function into its symmetric and antisymmetric parts. J. Algebraic Combin., 4(3):201-231, 1995.

[CM15] E. Carlsson and A. Mellit. A proof of the shuffle conjecture. arXiv:math/1508.06239, August 2015.

[GH03] A. Garsia and J. Haglund. A proof of the $q, t$-Catalan positivity conjecture. Adv. Math, 175:319-334, 2003. 
[GLWX15] A. M. Garsia, E. Leven, N. Wallach, and G. Xin. A new plethystic symmetric function operator and the Rational Compositional Shuffle Conjecture at $t=1 / q$. arXiv:1501.00631, January 2015.

[Hag04] J. Haglund. A proof of the $q, t$-Schröder conjecture. Internat. Math. Res. Notices, 11:525$560,2004$.

[Hag08] J. Haglund. The q,t-Catalan Numbers and the Space of Diagonal Harmonics. Amer. Math. Soc., 2008. Vol. 41 of University Lecture Series.

[Hai02] M. Haiman. Vanishing theorems and character formulas for the Hilbert scheme of points in the plane. Invent. Math., 149:371-407, 2002.

$\left[\mathrm{HHL}^{+}\right.$05] J. Haglund, M. Haiman, N. Loehr, J. B. Remmel, and A. Ulyanov. A combinatorial formula for the character of the diagonal coinvariants. Duke Math. J., 126:195-232, 2005.

[HRRW] J. Haglund, J. B. Remmel, B. Rhoades, and A. T. Wilson. Ordered set partition statistics and the Delta Conjecture. In preparation.

[LLT97] A. Lascoux, B. Leclerc, and J.-Y. Thibon. Ribbon tableaux, Hall-Littlewood functions, quantum affine algebras, and unipotent varieties. J. Math. Phys., 38(2):1041-1068, 1997.

[LW07] N. A. Loehr and G. S. Warrington. Square $q, t$-lattice paths and $\nabla\left(p_{n}\right)$. Trans. Amer. Math. Soc., 359(2):649-669, 2007.

[RW15] J. B. Remmel and A. T. Wilson. An extension of MacMahon's equidistribution theorem to ordered set partitions. J. Combin. Theory, Ser. A, 134:242-277, August 2015.

[Wil15a] A. T. Wilson. An extension of MacMahon's equidistribution theorem to ordered multiset partitions. arXiv:1508.06261, August 2015.

[Wil15b] A. T. Wilson. Generalized shuffle conjectures for the Garsia-Haiman delta operator. PhD thesis, UCSD, 2015.

[Wil15c] A. T. Wilson. A weighted sum over generalized Tesler matrices. arXiv:1510.02684, October 2015. 